\title{
Cognitive Predictors of Delay Discounting in Monetary Choices
}

\author{
Viera Bačová (iD), Jakub Šrol \\ Institute of Experimental Psychology, Centre of Social and Psychological Sciences, Slovak Academy of Sciences, \\ Bratislava, Slovak Republic
}

Delay discounting, the tendency to choose a smaller-sooner reward over a larger-later reward, has been conceptualized either as a personal preference or as a rational thinking component. In this study ( $N=$ 397), the associations between monetary delay discounting - constructed as a rational thinking task - and cognitive individual difference measures were examined. Participants with higher general cognitive ability, cognitive reflection, scientific reasoning, and objective numeracy had a weaker tendency to discount delayed rewards, the opposite was true for those with higher intuitive thinking disposition and bias susceptibility. Bias susceptibility predicted delay discounting over and above all other cognitive predictors. The results partially support the assumption about a common basis of delay discounting and susceptibility to cognitive biases (as a rational thinking indicator). Because of the relatively low explained variance in delay discounting by cognitive variables, however, ample room is left for other potential predictors in the monetary delay discounting tasks.

Key words: delay discounting, bias susceptibility, cognitive ability, individual differences

\section{Introduction}

Delay discounting ${ }^{1}$ refers to the reduction in

${ }^{1}$ As is well known, it is not uncommon in economics and psychology for different terms to be used for the same phenomenon. In the case of delay discounting, these are, for example, discount factor, discount rate, marginal private rate of discount, social discount rate, utility discount rate, intertemporal choice, pure discounting, time pref- the present value of an outcome as the postponing of that outcome rises (Kirby, 2009; Kirby \& Marakovic, 1996). The more remote a future outcome is, the lower its present val-

erence, subjective rate of time preference, impatience, time bias, temporal orientation, consumption rate of interest, time positivity inclination - to name at least some (Frederick, Loewenstein, \& O’Donoghue, 2002, list additional terms).

\footnotetext{
Correspondence concerning this article should be addressed to Viera Bačová, Institute of Experimental Psychology, Centre of Social and Psychological Sciences, Slovak Academy of Sciences, Dúbravská cesta 9, 84104 Bratislava, Slovak Republic. E-mail: viera.bacova@savba.sk

Supplementary material for this paper is available at https://journals.savba.sk/index.php/studiapsychologica/article/view/116/73
}

Received April 27, 2020 
ue for an individual, thus, the individual is less likely to choose the future outcome. Let us say we would offer you a choice between receiving a $50 €$ reward today or a $55 €$ one week later, which one would you choose? If you are like most people, chances are that you would ask yourself whether it is worth it to wait seven days for such a meager additional sum of money and rather choose to get the smaller amount right now without having to face the uncertainty. After all, who knows what will happen in the next seven days? Indeed, a vast body of research on discounting showed that people usually prefer the smaller-sooner to larger-later reward when it is offered immediately. Research and review studies (Frederick, Loewenstein, \& O'Donoghue, 2002; Read, 2004; Soman et al., 2005; Urminsky \& Zauberman, 2016) alike suggest that humans (and even animals) are generally impatient, often preferring smaller earlier rewards to larger delayed ones, even when waiting offers far higher utility than the choice of a near-term option.

However, individuals differ in how much they discount future rewards as a function of delay (Malesza \& Ostaszewski, 2017; Myerson, Baumann, \& Green, 2017). The stronger future outcomes discounting, the lower the present evaluation of future outcomes, and the higher the individual's tendency to choose a smaller-sooner outcome over a larger-later outcome. Why some people discount more and others less has been the subject of numerous research studies that have sought to shed light on the nature and mechanisms of this phenomenon. Our study adds to this research on individual differences as it tries to examine the underlying mechanisms of delay discounting by studying a wide range of cognitive predictors in the choices of present and future monetary rewards. Unlike most previous studies, we have included not only various cognitive abilities but also the susceptibility to cognitive biases as an indi- cator of rational thinking (Stanovich, West, \& Toplak, 2016) to explore the intertemporal choices and tendency to discount future monetary rewards. Before we introduce the present study in detail, we first would like to point out two distinctive ways in which delay discounting was conceptualized in previous research - either as a personal preference or as a rational thinking component. This is important because the study of the cognitive dimensions of delay discounting can be questioned by the objection that cognition plays a relatively weak role in delay discounting compared to personal time preferences.

\section{Delay Discounting as a Preference}

In terms of personality, delay discounting has been considered to be an expression of the desire for immediate or sooner gratification, and stronger delay discounting means succumbing to this desire (Kirby \& Finch, 2010; MacKillop et al., 2011). Thus, stronger delay discounting has become mostly accepted as an indicator of low self-control and willpower (Ainslie \& Haslam, 1992; Rachlin, 2000) that can ultimately induce a variety of personal and social maladies (Shenhav, Rand, \& Greene, 2017). Contrastingly, the choice of larger future rewards over smaller immediate rewards was found to be associated with many positive life outcomes, including better academic performance, healthier relationships, and improved social functioning (Mischel, Shoda, \& Rodriguez, 1989). Several studies examining the relationship between cognitive dispositions and delay discounting have been based on the comparisons of the relative strength of one's cognitive control networks over the emotional salience of the reward, and thus were neuro-psychologically oriented (Madden \& Bickel, 2010). Within a clinical context in psychology, the association of a stronger discounting with various forms 
of addictive behavior has been documented (Amlung et al., 2017).

A predominant number of discounting studies (including psychological ones) have been using measures based on economic models of delay discounting. The economic models assume that the value of a delayed reward is a function of the delay to that reward - where such variables as risk, uncertainty, and investment play a role - and the models seek to find the most precise expression of the function (Frederick et al., 2002; Read, 2004). Also, these models assume that the overweighing of short-term smaller gratification over the larger-later one is not a rational choice, as it violates the principle of maximizing the expected utility (a larger reward is always larger, even if it is necessary to wait for it). The action a rational person should choose is the one with the highest expected value regardless of the passage of time. For example, in monetary choices, the estimate of an annual interest rate could arguably be used to separate choices where one should wait relative to the remaining choices (Basile \& Toplak, 2015). However, economists admit that there are normatively acceptable reasons to devalue future outcomes, which are economic in nature, such as an anticipated increase in wealth over time, the possibility of intertemporal arbitrage, and the possibility of inflation.

\section{Delay Discounting as a Component of Ratio- nal Thinking}

In a similar vein, in recent rational thinking research, delay discounting began to be considered a component of instrumental rationality of an individual's goal fulfillment (Stanovich, 2011; Stanovich, West, \& Toplak, 2011). Consistently distinguishing rational thinking from intelligence, Stanovich and his colleagues have coined the term "rational temporal discounting" of future rewards (Stanovich, West, \& Toplak, 2016; Toplak, West, \& Stanovich, 2017). They include delay discounting tasks among indirect measures of avoiding or resisting intuitive information processing and thus important measures of rational thinking. Rational temporal discounting tasks are presented as the tasks that are dependent on "processing requirements" of rational thinking, i.e. conflict detection, inhibition of intuitive response, and hypothetical thinking necessary to calculate the correct answer. By doing so, they set delay discounting apart from other types of tasks that rely on "knowledge requirements", which depend on whether a person commands the numerical or logical rules needed to solve a task. In addition to delay discounting, this category of tasks includes a reflection versus intuition subtest, belief bias syllogisms, ratio bias, and disjunctive reasoning tasks (Stanovich et al., 2016).

In the tradition of the heuristics and biases approach, cognitive biases are identified as systematic deviations from the normative models of instrumental and epistemic rationality (Stanovich et al., 2016). They have been attributed to the use of intuitive heuristics, the rules of thumb, which people use to make quick and often accurate judgments and decisions, but which can lead to suboptimal reasoning under some circumstances. The current heuristics and biases research aims to understand the cognitive factors, which are related to the performance on various heuristics and biases tasks, and to produce new measurement instruments to study susceptibility to cognitive biases. In our study, we used the newly constructed battery of tasks of several frequently studied reasoning and decision-making biases whose psychometric properties have been tested in several studies (Šrol, 2021). 


\section{Present Research}

The distinction between general cognitive ability (intelligence) and rational thinking ability (avoidance of cognitive biases) has led us to explore the extent to which cognitive dispositions, which are related to rational thinking, influence people's tendency toward discounting future monetary rewards (Stanovich et al., 2016; Toplak et al., 2017; but see also Shenhav et al., 2017).

We have included a wide range of individual difference measures including cognitive ability, cognitive reflection, numeracy, and scientific reasoning, as well as self-reported analytic and intuitive thinking disposition. Several of these measures have already been explored in the context of delay discounting. The relation between cognitive ability and delay discounting appears to be confirmed, with better performance on measures of intelligence and cognitive control predicting weaker discounting rates (Shamosh \& Gray, 2008). Individuals who do not have sufficient cognitive resources are likely to favor smaller-sooner rewards. The stronger discounters were found to score lower on cognitive reflection (Shenhav et al., 2017). Białek and his colleagues (Białek, Bergelt, Majima, \& Koehler, 2019; Białek \& Sawicki, 2018) used numeracy and reinforcement sensitivity next to cognitive reflection to predict discounting. Yet, the remaining factors were not, to our knowledge, previously examined in this specific context, but they all have been shown to consistently contribute to the susceptibility to cognitive biases (e.g., Čavojová, Šrol, \& Jurkovič, 2020; Stanovich et al., 2016; Šrol, 2021), and are therefore considered important predictors of the general tendency toward rational thinking.

Our research question was not only whether impatience (in the form of stronger discounting of delayed monetary rewards) is related to traditionally researched cognitive abilities but whether there are also connections between delay discounting and the indicators of rational thinking, which Stanovich et al. (2016) explore as the capability not to succumb to cognitive biases. Therefore, we have included a separate measure of susceptibility to cognitive biases, to serve as an additional indicator of people's tendency toward intuitive processing, and to examine whether delay discounting is indeed related to the general proneness to cognitive biases.

\section{Monetary Choice Questionnaire}

To assess delay discounting, we chose the Monetary Choice Questionnaire (MCQ; Kirby \& Marakovic, 1996), which is considered "a behavioral economic measure of preference for smaller immediate rewards over larger delayed rewards and is also referred to as impulsive choice" (Mackillop et al., 2016, p. 3361). However, there are reasons in monetary choices to justify the choice of a smaller-earlier payment, such as anticipating high inflation in the future (for detailed discussion see Frederick et al., 2002). Therefore, we tried to eliminate these reasons for discounting future monetary outcomes by modifying the instructions in the questionnaire. We also sought to reduce the uncertainty of receiving rewards in the distant future by including assurance that the selected reward would be provided in the task instruction (see more in the Materials section). In doing so, we tried to eliminate all reasonable reasons for choosing a smaller reward and thus construct a task that could be considered a pure measure of "rational" delay discounting. In the terms of Frederick et al. (2002), we have sought not to assess 'time discounting' (which includes any reason for caring less about the future outcome) but to assess 'time preference' (which Frederick et al. refer to as the discounting 
future utility per se). According to Stanovich and his colleagues, the weak discounting in the modified questionnaire can be considered "rational" temporal discounting (Stanovich et al., 2016).

By including a wide range of general and specific cognitive abilities, thinking dispositions, as well as a bias susceptibility measure and the measure of pure preference of immediate or sooner monetary payments, we hope to disentangle those cognitive factors which play the most important role in delay discounting in monetary choices. We are looking for whether and in what way delay discounting is related to rational thinking both through its cognitive correlates and through a separate measure of rational thinking itself (bias susceptibility score).

\section{Method}

\section{Participants}

The analysis presented here is based on the data from a larger study reported by Šrol (2021) on the relationship between susceptibility to cognitive biases, endorsement of epistemically suspect beliefs, and cognitive individual difference predictors. Here, our focus lies solely on delay discounting understood as part of rational thinking, which was not analyzed in the previous study, and how it relates to cognitive abilities, thinking dispositions, and bias susceptibility. Participants were recruited through a Prolific online participant pool. The sample consisted of 397 participants $^{2}$ (140 men, 256 women, 1 genderqueer) with an average age of 36 years $(S D=12.47$, age range: $18-74)$. Post-hoc

\footnotetext{
${ }^{2}$ In total, 405 people participated in the study but based on attention check questions, eight participants were identified who either did not follow the instructions or showed patters of random responding and these were automatically excluded from all analyses.
}

sensitivity analysis conducted in G*Power 3.1 software (Faul, Erdfelder, Lang, \& Buchner, 2007) showed that the current sample size should provide good statistical power $(B=$ $.80)$ to detect any correlations of $r=.125$ and above with $5 \%$ type I error probability $(\alpha)$, as well as effects sizes of approximately $f^{2}=.02$ for a single coefficient in a regression analysis with 8 predictors.

\section{Materials}

The descriptive statistics and internal consistency estimates for all of the measures included in the present study are reported in Table 1.

Delay discounting. As a measure of delay discounting, we used the 27-item Money Choice Questionnaire taken from Kirby (2009). Every item presented participants with a choice between a smaller immediate reward and a larger delayed reward. To rule out various distinct considerations that underlie choices in delay discounting tasks, i.e. the factors that diminish the expected utility of a future consequence (uncertainty that the future reward will be obtained, expectations of changing utility, changing situations, etc.; see Frederick et al., 2002), we modified the task instructions in the Money Choice Questionnaire as follows: "When choosing from the alternatives please always assume with both options that you can be certain that you will receive the money, and that the offered sums from which you are about to choose would stay exactly the same. That is, even if you decide for the latter option you will not get any additional earnings or interests, just the amount which is specified in the option. Also, assume that all other relevant variables (i.e., political and economic situation, the value of money, your financial situation, and your attitude toward money) will not change in time, that they will all stay exactly the same as they are right now." We wanted to reduce the factors that diminish the expected utility of a future consequence as 
much as possible to gain a pure measure of "rational" delay discounting (i.e., time preference, according to Frederick et al., 2002), as opposed to time discounting, that is, discounting money for justified reasons (see above).

In line with the calculations described by Kirby (2009; Kirby \& Marakovic, 1996), we have estimated participants' overall delay discount rates $k$ from their choices using an automatic scorer developed by Kaplan, Lemley, Reed, and Jarmolowicz (2014). Since we were not interested in magnitude effects on delay discounting, we used the overall $k$ parameters rather than analyzing discounting rates for small, medium, and large rewards separately. As previous studies have done (e.g., Shenhav et al., 2017), we used the log10-transformed $k$ values rather than simple ones to correct for possible skewness in the distribution of discount rates. Also, we checked for consistency of the estimated $k$ parameters with participants' choices and we excluded three participants $(0.8 \%$ of the sample) whose consistencies were lower than $75 \%$ (see, for example, Kirby, 2009; Shenhav et al., 2017). We use these estimated log10-transformed overall $k$ parameters to measure people's temporal discounting ${ }^{3}$. Higher values of the $k$ parameter indicate a stronger tendency to discount future rewards.

\footnotetext{
${ }^{3}$ We have chosen to calculate $k$ parameter for every participant as a measure of delay discounting for consistency with some previous works in this domain (see, for example, Kirby, 2009; Shenhav et al., 2017). We are, however, aware that some previous studies adopted a simple scoring based on the choice of either smaller immediate or larger delayed reward to measure delay discounting rather than relying on the calculation of the $k$ parameter We have rerun all of the analyses below using the sum of choices for immediate smaller rewards as the measure of delay discounting (scoring every item of the Money Choice Questionnaire as 1 = choice of the smaller immediate reward, $0=$ choice of the larger delayed reward). The delay discounting measure calculated in this way was almost indistinguishable from the $k$ parameter values $(r=.99)$ and all of the analyses yielded results which are completely consistent with what is presented in the results section below.
}

General and specific cognitive abilities. We used five methods to assess general as well as specific cognitive abilities which may be related to delay discounting. The Vienna $\mathrm{Ma}$ trix Test (Klose, Černochová, \& Král, 2002) was used as a measure of general cognitive ability. The test originally consists of 24 items of increasing difficulty, which in their principle resemble Raven's progressive matrices. For the present study, we selected 14 items based on a pre-test (Šrol, 2019) to serve as a short cognitive ability measure. Further, we also employed measures to assess three more specific abilities: cognitive reflection, scientific reasoning ability, and numerical ability. The cognitive reflection measure consisted of ten problems that strongly elicited intuitive but incorrect answers. All problems were taken from previously used cognitive reflection measures (Šrol, 2019; Thomson \& Oppenheimer, 2016). Scientific reasoning ability was measured using Drummond's and Fischhoff's (2017) Scientific Reasoning Scale. The scale consists of 11 items with a true/false response format that tests people's understanding of the basic scientific concepts. To account for participants' competence at working with numerical information, we included: an objective numeracy measure, which consisted of eight problems taken from previous works (Cokely, Galesic, Schulz, Ghazal, \& Garcia-Retamero, 2012; Lipkus, Samsa, \& Rimer, 2001), which pertained to people's ability to work with information in various numerical formats, such as percentages and probabilities (example item: "If Person $A$ 's risk of getting a disease is 1 in 100 in ten years, and person B's risk is double that of A's, what is B's risk?"); and a short Subjective Numeracy Scale (McNaughton, Cavanaugh, Kripalani, Rothman, \& Wallston, 2015) in which participants were given three self-report items to evaluate their numerical abilities on a sixpoint scale (example item: "How good are you at working with fractions?"). 
Analytic and intuitive thinking disposition. A short, ten-item version of the Rational-Experiential Inventory taken from Epstein, Pacini, Denes-Raj, and Heier (Study 2; 1996) was used to tap participants' thinking dispositions. The inventory consists of a five-item Need for Cognition scale (example item: "I prefer complex to simple problems") intended to measure enjoyment of intellectual challenges and a general disposition toward analytic thought and a five-item Faith in Intuition scale (example item: "I believe in trusting my hunches") aimed at tapping the tendency to trust one's intuition and initial impressions. The items of both scales were presented in an intermixed order on a single page with the same five-point response scale on which participants rated the extent to which every item was characteristic of them. The average ratings calculated separately for the two scales were used as indicators of analytic and intuitive thinking disposition.

Bias susceptibility measure. To measure general susceptibility to cognitive biases, five frequently used tasks from the heuristics and biases research literature were employed: syllogistic reasoning with belief bias, baserate neglect, conjunction fallacy, ratio bias, and gambler's fallacy (e.g., Stanovich et al., 2016). All the heuristics and biases tasks that were included in the composite bias susceptibility score represent tasks that have heavy processing requirements and their successful solution indicates the avoidance of heuristic thinking (Stanovich, 2016). Most of the items were taken from materials used in previous studies on individual differences in susceptibility to cognitive biases (De Neys \& Franssens, 2009; Frey, Johnson, \& De Neys, 2018; Klaczynski, 2014; Toplak, Liu, Macpherson, Toneatto, \& Stanovich, 2007); some additional items were created by the authors to resemble these tasks. For every type of reasoning problem, there were six items, resulting in $\mathbf{3 0}$ items in total. Normatively correct responses on all of the problems were summed to create a single bias susceptibility composite. The composite score was then reversed so that higher values indicated higher susceptibility to cognitive biases.

\section{Results}

In the results, we firstly analyzed the correlations between participants' delay discounting rates and individual difference variables. Specifically, we wanted to see the patterns of correlations which the delay discounting rates exhibited with participants' general and specific cognitive abilities, self-reported numerical abilities and thinking dispositions, and the general susceptibility to cognitive biases. Secondly, we present the results of a hierarchical linear regression where all individual difference measures are examined as predictors of delay discounting.

\section{Correlations between Individual Difference Measures and Delay Discounting}

The correlations between individual difference cognitive variables and discounting rates are presented in Table 1. As can be seen from the table, the extent to which participants discounted delayed rewards was negatively associated with cognitive reflection, scientific reasoning, cognitive ability, and objective numeracy. The correlations were, however, relatively modest and ranged from small to moderate effect size. The most pronounced relationship was found in the case of cognitive reflection, which was already shown to be correlated with delay discounting to approximately the same extent by Shenhav et al. (2017). Interestingly, scientific reasoning, which, to our knowledge, has not been previously examined as a predictor of delay discounting, ended up correlating with par- 
ticipants' discounting rates to approximately the same extent as cognitive reflection. We also found that individuals with higher general cognitive ability have a weaker tendency to discount future rewards (Shamosh \& Gray, 2008). Finally, a weak correlation, albeit of similar size as in the case of other general and specific cognitive abilities, was found between delay discounting and objective numeracy. This suggests that the ability to work with numerical information does to some extent influence the decisions between the two offered rewards.

Concerning the thinking disposition variables, we found that while the self-reported tendency to process information intuitively was shown to relate to a stronger discounting of future rewards, there was no such relationship in the case of self-reported analytic thinking disposition. Importantly, however, overall bias susceptibility (i.e., the composite score on the five heuristics and biases tasks indicating the avoidance of miserly processing) has shown up to be the most pronounced correlate of the delay discounting. Unsurprisingly, the participants who were especially prone to fall prey to various biases in the battery of reasoning tasks were also the ones who tended to discount the future rewards to a stronger extent.

Considering the correlations among the cognitive predictors themselves, these were analyzed as a part of the larger study reported by Šrol (2021) from which the present analysis was drawn, and are scrutinized in more detail there. In short, the cognitive predictors exhibited patterns of relationships that were consistent with previous works on individual differences in susceptibility to cognitive biases (e.g., Čavojová, Šrol, \& Jurkovič, 2020; Klaczynski, 2014; Šrol \& De Neys, 2021; Stanovich, West, \& Toplak, 2016). That is, there were moderate to strong negative associations ( $r$ 's around .20 - .50) among cognitive ability, cognitive reflection, scientific reasoning, numeracy, and the susceptibility to cognitive biases. Self-reported intuitive and analytic thinking disposition, on the other hand, displayed weak to moderate correlations with the other variables ( $r$ 's around .10 - .30). As could be expected, bias susceptibility and intuitive thinking disposition were negatively related to variables that tapped the dispositions toward rational thinking (cognitive ability, cognitive reflection, scientific reasoning, numeracy, analytic thinking disposition).

Table 1 Descriptive statistics of variables used in the present study and their correlations with the delay discounting rate $(k)$

\begin{tabular}{|c|c|c|c|c|c|c|c|c|c|c|c|}
\hline \multicolumn{4}{|l|}{ Descriptives } & \multicolumn{8}{|c|}{ Correlations } \\
\hline & $M$ & $S D$ & $\alpha$ & 1. & 2. & 3. & 4. & 5. & 6. & 7. & 8. \\
\hline 1. Delay discounting $(k)$ & -1.99 & 0.72 & - & - & & & & & & & \\
\hline 2. Cognitive ability & 5.03 & 2.97 & .73 & -.22 & - & & & & & & \\
\hline 3. Cognitive reflection & 5.07 & 2.40 & .75 & -.25 & .48 & - & & & & & \\
\hline 4. Scientific reasoning & 6.99 & 2.52 & .68 & -.24 & .38 & .49 & - & & & & \\
\hline 5. Objective numeracy & 4.64 & 1.63 & .51 & -.18 & .40 & .50 & .38 & - & & & \\
\hline 6. Subjective numeracy & 3.96 & 1.16 & .79 & -.03 & .24 & .25 & .14 & .29 & - & & \\
\hline 7. Analytic thinking disposition & 3.42 & 0.78 & .78 & -.05 & .16 & .20 & .22 & .18 & .36 & - & \\
\hline 8. Intuitive thinking disposition & 3.81 & 0.68 & .84 & .20 & -.19 & -.25 & -.25 & -.17 & -.01 & -.04 & - \\
\hline 9. Bias susceptibility & 13.29 & 6.41 & .87 & .34 & -.37 & -.52 & -.49 & -.47 & -.23 & -.27 & .30 \\
\hline
\end{tabular}




\section{Cognitive Predictors of Delay Discounting: A Regression Analysis}

Since several variables theoretically related to rational thinking, as well as our bias susceptibility composite, have shown up to be correlated with the extent to which participants discounted future rewards, we have conducted a regression analysis to ascertain which of these factors independently predict delay discounting. We have entered all of the cognitive individual difference predictors along with bias susceptibility measure in a single step of the regression to explore their relative contributions to delay discounting. Besides the traditional linear regression results (regression coefficients and their statistical significance), we also present the results of dominance analysis conducted in yhat package of the $\mathrm{R}$ software (Nimon, Oswald, \& Roberts, 2020), which allowed us to examine the proportion of variance in delay discounting uniquely explained by each of our cognitive individual difference predictors. The summary of the regression analysis is shown in Table $2^{4}$.

The only reliable independent predictor in the regression ended up being bias susceptibility $(B=.25)$. That is, after statistically controlling for cognitive ability, cognitive reflection, scientific reasoning, objective and subjective numeracy, and thinking dispositions, bias susceptibility still predicted discounting rates to an approximately moderate extent. Its predictive power was, however, ob-

\footnotetext{
${ }^{4}$ Although the cognitive predictor variables were mutually correlated, there does not seem to be a problem with excessive multicollinearity - with all of the predictors included in the model, the largest VIF value was observed in the case of cognitive reflection (VIF $=1.793$ ). This value, however, is far smaller than the thresholds which are usually considered problematic, for example, Field (2013) suggests the threshold of VIF > 10 to indicate problems with excessive multicollinearity of the predictors in linear regression.
}

viously at the expense of other predictors in the analysis. While almost all of the cognitive individual difference variables were correlated with delay discounting (Table 1), after their simultaneous inclusion in the model, none of the factors besides bias susceptibility significantly predicted discounting rates. Besides, the cognitive individual difference predictors together explained $12 \%$ of the variance in delay discounting, leaving ample room for other potential predictors in this regard.

In order to examine the relative importance of cognitive individual difference variables in predicting people's delay discounting rates, we have also calculated general dominance statistics for every predictor in the regression. The general dominance (GD) values reported in Table 2 represent the proportion of variance in delay discounting uniquely explained by each predictor in the model. As can be seen from the table, the highest proportion of variance (approximately 6\%) in delay discounting is explained by bias susceptibility. Interestingly, while cognitive ability, cognitive reflection, scientific reasoning, and intuitive thinking were all non-significant predictors in the regression model, each of them contributed around 2\% of the variance in delay discounting. Still, it should be noted that the overall proportion of explained variance in delay discounting is surprisingly low given the extensive amount of cognitive individual difference predictors employed in the present study.

The sample in our study came from a very broad age range. This could have affected the results since age has been shown to be reliably associated with some of the cognitive individual difference variables employed in the present study, such as cognitive ability. For this reason, we have repeated our key regression analysis while including the age in the model to see whether its inclusion will lead to any changes in patterns of observed results, as well as to examine whether age can explain 
Table 2 Summary of the regression analysis predicting the delay discounting rate ( $k$ )

\begin{tabular}{lccccc}
\hline & $b(S E)$ & $b$ & $t$ & $p$ & GD \\
\hline Delay discounting & & & & & \\
Constant & $-2.67(0.33)$ & & -8.09 & $<.001$ & \\
Cognitive ability & $-0.02(0.01)$ & -.09 & -1.52 & .128 & .016 \\
Cognitive reflection & $-0.02(0.02)$ & -.06 & -0.96 & .337 & .019 \\
Scientific reasoning & $-0.02(0.02)$ & -.06 & -0.94 & .346 & .018 \\
Objective numeracy & $0.01(0.03)$ & .02 & 0.31 & .759 & .008 \\
Subjective numeracy & $0.03(0.03)$ & .05 & 0.97 & .335 & .002 \\
Analytic thinking disposition & $0.03(0.05)$ & .04 & 0.72 & .473 & .001 \\
Intuitive thinking disposition & $0.09(0.05)$ & .08 & 1.66 & .098 & .016 \\
Bias susceptibility & $0.03(0.01)$ & .25 & 4.07 & $<.001$ & .061 \\
\multicolumn{1}{c}{$R^{2}=.123, F(8,385)=7.89, p<.001$} & & & & \\
\hline
\end{tabular}

Note. The table contains unstandardized $(b)$ and standardized regression coefficients $(b)$ with their respective $t$-ratio and significance. $R^{2}$ denotes adjusted $r$-square for the initial model with appropriate model statistics. Significant regression coefficients $(p<.05)$ are presented in italics. The column labeled GD gives the proportion of variance in the delay discounting uniquely explained by each predictor in the regression, as calculated from the dominance analysis conducted in the $R$ package yhat (Nimon, Oswald, \& Roberts, 2020).

any additional variance in delay discounting above the cognitive individual difference predictors. The results of this analysis are presented in the Supplementary Materials. In short, age showed up as a significant independent predictor $(B=-.15)$ and its inclusion in the model lead to a further $2 \%$ explained variance in delay discounting. Interestingly, after the differences in participants' age have been statistically controlled for, cognitive ability emerged as a significant, albeit relatively weak $(B=-.11)$ predictor of delay discounting as well. Both age and cognitive ability were negatively related to the strength to which participants discounted future rewards.

\section{Discussion}

As Stanovich (2018) explains, one of the defaults that evolution has made is to consume resources immediately, because it gives us a greater chance of surviving in an uncertain and hostile environment. However, in an environment in which most people live today, this default is not useful and no longer effective. In stable conditions, the normative answer in delay discounting tasks should be to maximize the expected utility at least in certain types of rewards. Overcoming an evolutionary default in a reasonable wait for remuneration, however, requires the involvement of analytic cognitive processes which presuppose expending cognitive effort. In our research, we examined if and how the tendency to discount future monetary rewards is related to various cognitive predictors to disentangle the cognitive roots of delay discounting. More specifically, we focused on exploring delay discounting in monetary choices with predictors traditionally associated with rational thinking and, at the same time, with a novel general measure of proneness to cognitive biases.

As expected based on previous research, cognitive reflection and cognitive ability have 
shown up to correlate with delay discounting (e.g., Basile \& Toplak, 2015; Shenhav et al., 2017). While we are not aware of a study that would examine the relationship between scientific reasoning and temporal discounting, the result is consistent with previous research, which confirmed scientific reasoning as an important predictor of bias susceptibility (Čavojová et al., 2020; Šrol, 2021). The higher numeracy was related to weaker discounting of future outcomes, as could be expected on the basis of the numerical nature of the task. Although the thinking dispositions have been considered to play an important role in rational thinking, past research has found relatively low or insignificant correlations between temporal discounting and various analytic thinking dispositions, such as the need for closure or actively open-minded thinking (e.g., Shenhav et al., 2017; Stanovich et al., 2016). Similarly, in our results, we have found a weak positive correlation between delay discounting and self-reported intuitive thinking, however, no relationship was found between delay discounting and self-reported analytic thinking.

The most pronounced correlate of the temporal discounting ended up being the overall bias susceptibility. Importantly, we have shown in the present study that the bias susceptibility was the only variable that predicted delay discounting in the regression analysis where all other variables were accounted for. This result shows that delay discounting has important associations with other tasks from the heuristics and biases literature and can be (at least partially) conceived of as a measure of rational thinking (Stanovich et al., 2016). It also explains why other cognitive individual difference variables did not show up to predict delay discounting along with the bias susceptibility measure independently. That is, intuitive thinking disposition, cognitive ability, or cognitive reflection reflect the processing requirements for recognizing and suppressing the intuitive heuristic response, which is a necessary component of most rational thinking tasks (Stanovich, 2011; Stanovich et al., 2016). For that reason, after including bias susceptibility measure as a predictor of delay discounting, none of the other individual difference variables were related to the tendency to discount future rewards.

And yet, it should be noted that the relatively low proportion of explained variance in delay discounting in the present study - the regression model lead to $12 \%$ of explained variance - is in sharp contrast with the typical results obtained in studies on individual differences in susceptibility to cognitive biases. Usually, studies indicate that several variables - cognitive ability, thinking dispositions, cognitive reflection, and numeracy-independently predict susceptibility to cognitive biases and explain around $20-40 \%$ of the variance, depending on which specific cognitive predictors are included and on the particular tasks used to measure the susceptibility to cognitive biases (Čavojová et al., 2020; Klaczynski, 2014; Šrol, 2021; Šrol \& De Neys, 2021; Stanovich et al., 2016). That is, while we showed support for the link between delay discounting and susceptibility to a wider range of cognitive biases, cognitive individual difference variables included in our study do not seem to be the only, or even the most important, predictors of delay discounting. This point is particularly pronounced when we consider that in our study, we included almost all of the variables which are traditionally thought to contribute the most to rational thinking.

It can be reasonably assumed that the delay discounting phenomenon is not determined or influenced by a single factor (or the only dimension of mental functioning) and that there are plausibly multiple contributors responsible for the phenomenon. More or less empirically verified hypotheses are based on 
people's relationship to the future (uncertainty about the future, time preference), perception of the present and future events (construal level theory), personality (e.g., optimism, impulsivity, self-control), and relationship to self (intrapersonal empathy gap) (Soman et al., 2005). Some evidence indicates that delay discounting is sensitive to both state and trait influences, however, little evidence has been provided as to whether delay discounting could be considered a personality trait (Odum, 2011).

In this research, we focused on the role of cognitive factors that underlie the performance on monetary choice tasks framed from the rational thinking perspective, i.e. tasks that were designed so that the rational choice was not to discount larger-later rewards. We have observed relatively low explained variance in our regression that examined the relative proportions of cognitive factors in delay discounting even though we have included most of the traditionally studied predictors of rational thinking (e.g., Šrol, 2021). This suggests that there are other factors/dimensions that may be much more important in delay discounting and play a greater role in monetary choices as well. Perhaps, future research could include personality and other variables as well as cognitive predictors of rational thinking to disentangle their relative roles in the tendency to discount future rewards in the monetary choice task.

As the benefit of this study, we consider the finding that among the tests of cognitive abilities, the susceptibility to cognitive biases showed up as the most important predictor of people's tendency to discount future monetary rewards. This indicates that delay discounting shares some common ground with the tasks studied in the heuristics and biases literature, which tap the ability to detect and inhibit the tendency for intuitive information processing (Stanovich et al., 2016).

\section{Acknowledgement}

The study was supported by the Slovak Research and Development Agency as part of the research project APVV-16-0153: "Cognitive failures - individual predictors and intervention possibilities" and by the scientific grant agency of the Ministry of Education, Science, Research and Sport of the Slovak Republic as part of the project VEGA 2/0053/21: "Examining unfounded beliefs about controversial social issues". We would like to thank Michał Białek and two anonymous reviewers for their helpful comments on an earlier version of this manuscript.

\author{
Authors' ORCID \\ Viera Bačová \\ https://orcid.org/0000-0002-3661-208X \\ Jakub Šrol \\ https://orcid.org/0000-0002-1168-2639
}

\section{References}

Ainslie, G., \& Haslam, N. (1992). Self-control. In G. Loewenstein \& J. Elster (Eds.), Choice over time (pp. 57-92). New York: Russell Sage Foundation. Amlung, M., Vedelago, L., Acker, J., Balodis, I., Mackillop, J. (2017). Steep delay discounting and addictive behavior: A meta-analysis of continuous associations. Addiction, 112(1), 51-62. https://doi.org/10.1111/add.13535

Basile, A. G., \& Toplak, M. E. (2015). Four converging measures of temporal discounting and their relationships with intelligence, executive functions, thinking dispositions, and behavioral outcomes. Frontiers in Psychology, 6, 13. https:// doi.org/10.3389/fpsyg.2015.00728

Białek, M., Bergelt, M., Majima, Y., \& Koehler, D. J. (2019). Cognitive reflection but not reinforcement sensitivity is consistently associated with delay discounting of gains and losses. Journal of Neuroscience, Psychology, and Economics, 12(3-4), 169.

Białek, M., \& Sawicki, P. (2018). Cognitive reflection effects on time discounting. Journal of In- 
dividual Differences, 39(2), 99-106. https://doi. org/10.1027/1614-0001/a000254

Čavojová, V., Šrol, J., \& Jurkovič, M. (2020). Why should we try to think like scientists? Scientific reasoning and susceptibility to epistemically suspect beliefs and cognitive biases. Applied Cognitive Psychology, 34(1), 85-95. https://doi. org/10.1002/acp.3595

Cokely, E. T., Galesic, M., Schulz, E., Ghazal, S., \& Garcia-Retamero, R. (2012). Measuring risk literacy: The Berlin Numeracy Test. Judgment and Decision Making, 7(1), 25-47.

De Neys, W., \& Franssens, S. (2009). Belief inhibition during thinking: Not always winning but at least taking part. Cognition, 113(1), 45-61. https://doi: 10.1016/i.cognition.2009.07.009

Drummond, C., \& Fischhoff, B. (2017). Development and validation of the Scientific Reasoning Scale. Journal of Behavioral Decision Making, 30(1), 2638. https://doi.org/10.1002/bdm.1906

Epstein, S., Pacini, R., Denes-Raj, V., \& Heier, H. (1996). Individual differences in intuitive-experiential and analytical-rational thinking styles. Journal of Personality and Social Psychology, 71(2), 390-405.

Faul, F., Erdfelder, E., Lang, A.-G., \& Buchner, A. (2007). G*Power 3: A flexible statistical power analysis program for the social, behavioral, and biomedical sciences. Behavioral Research Methods, 39(2), 175-191. https://doi.org/10.3758/ BF03193146

Field, A. (2013). Discovering statistics using IBM SPSS statistics (4th ed.). London: Sage.

Frederick, S., Loewenstein, G., \& O'Donoghue T. (2002). Time discounting and time preference: A critical review. Journal of Economic Literature, 40(2), 351-401. https://doi.org/ 10.1257/002205102320161311

Frey, D., Johnson, E. D., \& De Neys, W. (2018). Individual differences in conflict detection during reasoning. Quarterly Journal of Experimental Psychology, 71(5), 1188-1208. https://doi.org/1 $\underline{0.1080 / 17470218.2017 .1313283}$

Kaplan, B. A., Lemley, S. M., Reed, D. D., \& Jarmolowicz, D. P. (2014). 21- and 27-Item Monetary Choice Questionnaire Automated Scorers. Retrieved from https://kuscholarworks.ku.edu/ handle/1808/15424

Kirby, K. N. (2009). One-year temporal stability of delay-discount rates. Psychonomic Bulletin \&
Review, 16(3), 457-462. https://doi:10.3758/ PBR.16.3.457

Kirby, K. N., \& Finch, J. (2010). The hierarchical structure of self-reported impulsivity in a college-age population. Personality and Individual Differences, 48(6), 704-713. https://doi.org/10.1016/i.paid.2010.01.019

Kirby, K. N., \& Marakovic, N. N. (1996). Delay-discounting probabilistic rewards: Rates decrease as amounts increase. Psychonomic Bulletin \& Review, 3, 100-104. https://doi.org/10.3758/BF03210748

Klaczynski, P. A. (2014). Heuristics and biases: Interactions among numeracy, ability, and reflectiveness predict normative responding. Frontiers in Psychology, 5, 1-13. https://doi.org/10.3389/ fpsyg.2014.00665

Klose, J., Černochová, D., \& Král, P. (2002). Vídeňský Maticový Test. Praha: Testcentrum.

Lipkus, I. M., Samsa, G., \& Rimer, B. K. (2001). General performance on a numeracy scale among highly educated samples. Medical Decision Making: An International Journal of the Society for Medical Decision Making, 21(1), 37-44. https:// doi.org/10.1177/0272989X0102100105

MacKillop, J., Amlung, M. T., Few, L. R., et al. (2011). Delayed reward discounting and addictive behavior: $\mathrm{A}$ meta-analysis. Psychopharmacology, 216, 305-321. https://doi.org/10.1007/s00213-011-2229-0

MacKillop, J., Weafer, J., Gray, J. C., Oshri, A., Palmer, A., \& de Wit, H. (2016). The latent structure of impulsivity: Impulsive choice, impulsive action, and impulsive personality traits. Psychopharmacology, 233, 3361-3370. https://doi.org/10.1007/s00213016-4372-0

Madden, G. J., \& Bickel, W. K. (Eds.). (2010). Impulsivity: The behavioral and neurological science of discounting. American Psychological Association. https://doi.org/10.1037/12069-000

Malesza, M., \& Ostaszewski, P. (2017). Assessing individual differences in discounting: Construction and initial validation of the Discounting Inventory. Current Psychology, 39, 207-219. https://doi. org/10.1007/s12144-017-9754-x

McNaughton, C. D., Cavanaugh, K. L., Kripalani, S., Rothman, R. L., \& Wallston, K. A. (2015). Validation of a short, 3-Item version of the Subjective Numeracy Scale. Medical Decision Making, 35(8), 932936. https://doi.org/10.1177/0272989X15581800

Mischel, W., Shoda, Y., \& Rodriguez, M. L. (1989). Delay of gratification in children. Science, 
244(4907), 933-938. https://doi.org/10.1126/ science. 2658056

Myerson, J., Baumann, A. A., \& Green, L. (2017). Individual differences in delay discounting: Differences are quantitative with gains, but qualitative with losses. Journal of Behavioral Decision Making, 30(2), 359-372. https://doi.org/10.1002/ bdm.1947

Nimon, K., Oswald, F., \& Roberts, J. K. (2020). yhat: Interpreting Regression Effects [computer software]. Retrieved from https://cran.r-project. org/web/packages/yhat/yhat.pdf

Odum, A. L. (2011). Delay discounting: Trait variable? Behavioural Processes, 87(1), 1-9. https:// doi.org/10.1016/i.beproc.2011.02.007

Rachlin, H. (2000). The science of self-control. Cambridge, MA: Harvard University Press.

Read, D. (2004). Intertemporal choice. In D. J. Koehler \& N. Harvey (Eds.), Blackwell Handbook of Judgment and Decision Making ( $p$. 424-443). Blackwell Publishing. https://doi. org/10.1002/9780470752937.ch21

Shamosh, N. A., \& Gray, J. (2008). Delay discounting and intelligence: A meta-analysis. Intelligence, 36(4), 289305. https://doi.org/10.1016/i.intell.2007.09.004

Shenhav, A., Rand, D. R., \& Greene, J. D. (2017). The relationship between intertemporal choice and following the path of least resistance across choices, preferences, and beliefs. Judgment and Decision Making, 12(1), 1-18.

Soman, D., Ainslie, G., Frederick, S., Li, X., Lynch, J., Moreau, P., Mitchell, A., Read, D., Sawyer, A., Trope, Y., Wertenbroch, K., \& Zauberman, G. (2005). The psychology of intertemporal discounting: Why are distant events valued differently from proximal ones? Marketing Letters, 16(3-4), 347-360. http:// hdl.handle.net/10.1007/s11002-005-5897-x

Stanovich, K. E. (2011). Rationality and the reflective mind. New York: Oxford University Press.

Stanovich, K. E. (2016). The comprehensive assessment of rational thinking. Educational Psychologist, 51(1), 23-34. https://doi.org/10.1080/0046 $\underline{1520.2015 .1125787}$

Stanovich, K. E. (2018). Miserliness in human cognition: The interaction of detection, override and mindware. Thinking and Reasoning, 24(4), 423-444. https://doi.org/10.1080/13546783.20 18.1459314

Stanovich, K. E., West, R. F., \& Toplak, M. E. (2011). Individual differences as essential components of heuristics and biases research. In K. Manktelow, D. E. Over, \& S. Elqayam (Eds.), The Science of Reason: A Festschrift for Jonathan St. B. T. Evans. (pp. 355-396). Hove: Psychology Press.

Stanovich, K. E., West, R. F., \& Toplak, M. E. (2016). The Rationality Quotient: Toward a test of rational thinking. MIT Press.

Šrol, J. (2019). Individual differences in susceptibility to cognitive biases: Implications for theories of rational thought (Unpublished doctoral thesis). Bratislava.

Šrol, J. (2021). Individual differences in epistemically suspect beliefs: The role of susceptibility to cognitive biases. https://psyarxiv.com/4jcf7/

Šrol, J., \& De Neys, W. (2021) Predicting individual differences in conflict detection and bias susceptibility during reasoning. Thinking \& Reasoning, 27(1), 38-68. https://doi.org/10.1080/1354678 $\underline{3.2019 .1708793}$

Thomson, K. S., \& Oppenheimer, D. M. (2016). Investigating an alternate form of the cognitive reflection test. Judgment and Decision Making, 11(1), 99-113.

Toplak, M. E., Liu, E., Macpherson, R., Toneatto, T., \& Stanovich, K. E. (2007). The reasoning skills and thinking dispositions of problem gamblers: A dual-process taxonomy. Journal of Behavioral Decision Making, 20(2), 103-124. https://doi. org/10.1002/bdm.544

Toplak, M. E., West, R. F., \& Stanovich, K. E. (2017). Real-world correlates of performance on heuristics and biases tasks in a community sample. Journal of Behavioral Decision Making, 30(2), 541-554. https://doi.org/10.1002/bdm.1973

Urminsky, O., \& Zauberman, G. (2016). The psychology of intertemporal preferences. In G. Keren \& G. Wu (Eds.), The Wiley Blackwell Handbook of Judgment and Decision Making (pp. 141-181). John Wiley \& Sons, Chichester, UK. 